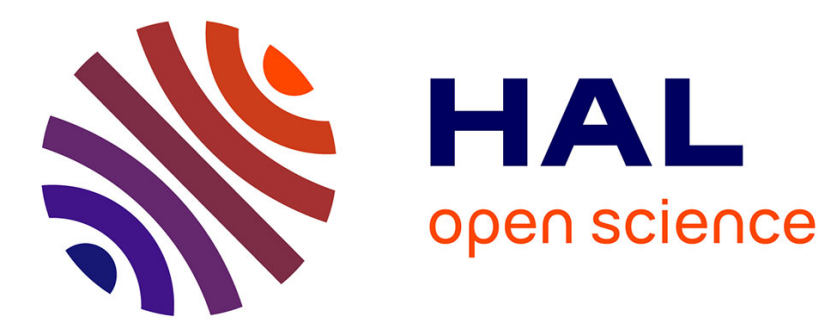

\title{
Wave Mixing and Electromagnetic Wave Scattering in Plasmas
}

\author{
A. Sitenko
}

\section{To cite this version:}

A. Sitenko. Wave Mixing and Electromagnetic Wave Scattering in Plasmas. Journal de Physique IV Proceedings, 1995, 05 (C6), pp.C6-7-C6-12. 10.1051/jp4:1995602 • jpa-00253965

\section{HAL Id: jpa-00253965 https://hal.science/jpa-00253965}

Submitted on 1 Jan 1995

HAL is a multi-disciplinary open access archive for the deposit and dissemination of scientific research documents, whether they are published or not. The documents may come from teaching and research institutions in France or abroad, or from public or private research centers.
L'archive ouverte pluridisciplinaire HAL, est destinée au dépôt et à la diffusion de documents scientifiques de niveau recherche, publiés ou non, émanant des établissements d'enseignement et de recherche français ou étrangers, des laboratoires publics ou privés. 


\title{
Wave Mixing and Electromagnetic Wave Scattering in Plasmas
}

\author{
A.G. Sitenko \\ Bogolyubov Institute for Theoretical Physics, National Ukrainian Academy of Sciences, \\ Metrolohichna 14b, 252143 Kiev, Ukraine
}

\begin{abstract}
Kinetic and fluid treatments of nonlinear wave interactions (wave mixing and electromagnetic wave scattering) in plasmas are considered and the difference between the two approaches is discussed. Solving the kinetic equation by the method of moments makes it possible to substantiate the validity and find the applicability range of the fluid approach to the study of nonlinear plasma processes. In particular, the detailed study of nonlinear processes in a plasma with external magnetic fields is performed. In the case of wave transformation, when mixed waves satisfy linear wave equations, both fluid and kinetic approaches yield similar expressions for the inducing current. In the case of electromagnetic wave scattering by thermal fluctuations, the results of the tluid and kinetic approaches are in accordance only in the limiting case of cold plasmas when thermal effects may be completely disregarded (these are thermal corrections to the wave dispersion equation and the additional Langevin source in the constitutive equation for fluctuations). Different expressions for the inducing current, obtained from various versions of hydrodynamic equations, reduce to the same form in the cold plasma limiting case.
\end{abstract}

\section{INTRODUCTION}

Electromagnetic wave scattering is a well known method of plasma diagnostics both in experimental fusion devices and near-Earth space environment. It is peculiar of the electromagnetic wave scattering in plasmas that along with Thomsom scattering - incoherent scattering by individual particles - there occurs as well scattering by collective plasma excitations - the combination scattering. The phenomenon of electromagnetic wave combination scattering and transformation by collective plasma excitations was considered for the first time in Ref. [1]. A detailed theory of electromagnetic wave scattering in plasmas with and without external fields was developed in papers [2-5] and, independently, in [6-8].

In view of extensive studies of plasma dynamics in fusion devices, much interest has been attracted to the calculations of electromagnetic wave scattering spectra for nonequilibrium plasmas with strong magnetic fields. A series of such calculations were performed in Refs. [9-14]. Some of them ([12-14]) doubt the hydrodynamic approach applicability to the treatment of nonlinear effects even in cold plasmas. In paper [15] we showed that the reasoning of [13,14] concerning the inapplicability of the fluid approximation for the study of nonlinear phenomena in plasmas had been erroneous. We considered a simple case of two-wave interaction in a plasma without external magnetic field to illustrate the identity of the results obtained by means of kinetic and fluid approaches in the limiting case of cold plasmas. Insofar as the approach in terms of hydrodynamic equations considerably simplifies the treatment of nonlinear processes occurring in a plasma, it is a matter of principle to substantiate the fluid approximation applicability and to reveal the relation between the results obtained by kinetic and fluid approaches. The problem is considered in this paper.

\section{ELECTROMAGNETIC FIELD IN A MEDIUM}

The study of electromagnetic field in any medium implies solving Maxwell's equations along with constitutive equations which describe the self-consistent interaction of component charged particles and determine the relationship between the current and the electric field in the medium, 


$$
\vec{j}=\vec{j}(\vec{E})+\vec{j}_{\mathrm{ext}}
$$

where $\vec{j}_{\text {ext }}$ is the external current. The general relation between the current $\vec{j}$ and the electric field $\vec{E}$ is nonlinear. If the field is weak, this relation may be written as an expansion in power series of the field, i.e.,

$$
\overrightarrow{j(E)}=\frac{\partial}{\partial t}\left(\hat{\varkappa} \vec{E}+\hat{\varkappa}^{(2)} \vec{E} \vec{E}+\ldots\right)
$$

where $\hat{x}$ and $\hat{x}^{(2)}$ are the linear and the nonlinear quadratic electric susceptibilities of the plasma. Taking into account the quadratic term in the expansion (2) provides, in particular, a possibility to describe the wave mixing.

If expansion $(2)$ is restricted to the linear term, i.e., $\vec{j}(\vec{E})=-i \omega \hat{x} \vec{E}$,

then the field in the homogeneous stationary medium is described by the usual Maxwell equation

$$
\hat{\Lambda}(\omega, \vec{k}) \vec{E}_{\vec{k} \omega}=-\frac{i}{\omega} \vec{j}_{\text {ext }}
$$

with the tensor $\hat{\Lambda}(\omega, \vec{k})$ being a function of the dielectric permittivity $\hat{\varepsilon}$ of the medium, i.e.,

$$
\Lambda_{i j}(\omega, \vec{k})=\varepsilon_{i j}(\omega, \vec{k})+\left(\frac{k_{i} k_{j}}{k^{2}}-\delta_{i j}\right) \eta^{2}, \quad \eta^{2}=\frac{k^{2} c^{2}}{\omega^{2}} \text {. }
$$

With regard for the fluctuations of the medium parameters responsible for its electromagnetic properties (charged particle and current densities, external magnetic field), the linear relation (3) reduces to

$\vec{j}(\vec{E})=-i \omega \hat{x} \vec{E}-i \omega \delta \hat{x} \vec{E}$

where $\delta \hat{x}$ is the fluctuation addition to the linear susceptibility tensor. Then the electromagnetic field in the medium with no external currents $\left(j_{\text {ext }}=0\right)$ is described by an equation of the type (4), with $j_{\text {ext }}$ being replaced by the current

$$
\vec{J} \equiv-i \omega \delta \hat{\varkappa} \vec{E}
$$

that is produced by electromagnetic field interaction with fluctuations. This interaction can give rise to the scattering of electromagnetic waves.

Assuming the fluctuations to be small, one can easily describe the electromagnetic wave scattering in terms of the perturbation theory. Suppose that, in the case of no fluctuations, an electromagnetic wave $\vec{E}_{0}$ with the frequency $\omega_{0}$ and the wave vector $\vec{k}_{0}$ propagates in the medium, i.e.,

$$
\Lambda_{i j}\left(\omega_{0}, \vec{k}_{0}\right) E_{j}^{0}=0
$$

Interaction of this wave with the fluctuations produces the field $\vec{E}$ of scattered waves with frequencies $\omega$ and wave vectors $\vec{k}$ that is governed by the equation

$$
\hat{\Lambda}(\omega, \vec{k}) \vec{E}_{\vec{k} \omega}=-\frac{i}{\omega} \vec{J}_{\vec{k} \omega}, \quad \vec{J}_{\vec{k} \omega}=-i \omega \delta x(\Delta \omega, \vec{q}) \vec{E}_{0}
$$

where $\Delta \omega=\omega-\omega_{0}, \vec{q}=\vec{k}-\vec{k}_{0}$. If fluctuations do not depend on the self-consistent electromagnetic interaction of medium particles, then there occurs incoherent wave scattering. The incoherent fluctuation spectrum contains a broad maximum for low frequencies $\Delta \omega$ whose width is determined by the temperature. Thus, the incoherent wave scattering is accompanied by small frequency shifts.

The self-consistent interaction of medium particles produces coherent fluctuations (collective fluctuation excitations) associated with sharp maxima in the spectrum at eigenfrequencies $\omega_{\vec{k}}$. Inasmuch as in this case the addition $\delta x$ depends on the fluctuation field (is proportional to the field), the inducing current in (9) is quadratic with respect to the field. Therefore, the wave scattering by collective fluctuations may be regarded as mixing of the incident wave $\left(\omega_{0}, k_{0}\right)$ and the fluctuation wave $\left(\Delta \omega=\omega_{\vec{q}}, \vec{q}\right)$, accompanied by the appearance of the wave $(\omega, \vec{k})$.

\section{REDUCTION OF THE KINETIC EQUATION TO THE CHAIN OF MOMENT EQUATIONS}

The main task in calculating the cross-sections of wave scattering or mixing is to derive from the constitutive equation an expression for the current given rise to by the incident wave 
nonlinear interaction with fluctuations, and determines the scattered wave field. The nonlinear constitutive equation for the plasma, and hence the scattered-wave-inducing current, may be derived from kinetic or hydrodynamic equations for the electron and ion plasma components. Since the difference between electron and ion masses is very large, the consideration may be restricted to the electron component only. The relevant kinetic equation may be written as

$$
\frac{\partial F}{\partial t}+\vec{v} \frac{\partial F}{\partial \vec{r}}+\frac{e}{m}\left(\vec{E}+\frac{1}{c}\left[\vec{v}, \vec{B}_{0}+\vec{B}\right]\right) \frac{\partial F}{\partial \vec{v}}=0
$$

where $F(\vec{v})$ is the electron distribution function, $\vec{B}_{0}$ is the external magnetic field, $\vec{E}$ and $\vec{B}$ are self-consistent electric and magnetic fields. The kinetic equation (10) is nonlinear with regard for the self-consistent electron interaction.

In order to compare the results obtained by kinetic and hydrodynamic approaches, we solve the kinetic equation by the method of moments. Then nonlinear equation (10) reduces to an infinite chain of equations for the distribution function moments

$$
\begin{aligned}
& \frac{\partial N}{\partial t}+\frac{\partial V_{i}}{\partial r_{i}}=0, \quad \frac{\partial V_{i}}{\partial t}+\frac{\partial V_{i j}}{\partial r_{j}}=\frac{e}{m}\left\{N E_{i}+\frac{1}{c} \epsilon_{i j k} v_{j}\left(B_{0 k}+B_{k}\right)\right\}, \\
& \frac{\partial V_{i j}}{\partial t}+\frac{\partial V_{i j k}}{\partial r_{k}}=\frac{e}{m}\left\{V_{i} E_{j}+E_{i} v_{j}+\frac{1}{c}\left(\in_{i k l} v_{j k}+\in_{j k l} v_{i k}\right)\left(B_{0 l}+B_{l}\right)\right\}
\end{aligned}
$$

and so on, where $N=\int d \vec{v} F$ is the electron density, $v_{i}=\int d \vec{v} v_{i} F$ is the electron flux density, $v_{i j}=\int d \vec{v} v_{i} v_{j} F$ and $v_{i j k}=\int d \vec{v} v_{i} v_{j} v_{k} F$ are the second- and third-order moments. We note that

$$
v_{i j}=\frac{v_{i} v_{j}}{N}+\Delta_{i j}
$$

where $\Delta_{i j}$ is the pressure tensor given by

$$
\Delta_{i j}=\int d \vec{v}\left(v_{i}-\frac{v_{i}}{N}\right)\left(v_{j}-\frac{v_{j}}{N}\right) F .
$$

With no heat fluxes, the chain of equations (11) is cut off by virtue of the relation

$$
v_{i j k}=\frac{1}{N^{2}} v_{i} v_{j} v_{k}+\frac{1}{N}\left(\Delta_{i j} v_{k}+\Delta_{i k} v_{j}+\Delta_{j k} v_{i}\right)
$$

Having substituted (14) into the third equation of the set (11), we obtain an equation for the quantity $\Delta_{i j}$, i.e.,

$$
\begin{aligned}
& \frac{\partial \Delta_{i j}}{\partial t}-\frac{e}{m c}\left(\epsilon_{i k l} \Delta_{j k}+\epsilon_{j k l} \Delta_{i k}\right) B_{0 t}= \\
& =\frac{e}{m c}\left(\epsilon_{i k l} \Delta_{j k}+\epsilon_{j k l} \Delta_{i k}\right) B_{l}-\frac{\Delta_{i j}}{N} \operatorname{div} \vec{v}-(\vec{v} \nabla) \frac{\Delta_{i j}}{N}-\Delta_{i k} \frac{\partial}{\partial r_{k}}\left(\frac{v_{j}}{N}\right)-\Delta_{j k} \frac{\partial}{\partial r_{k}}\left(\frac{v_{i}}{N}\right)
\end{aligned}
$$

(for the sake of convenience we moved all the nonlinear terms into the right-hand part). The plasma current $\vec{j}$ is immediately determined by the electron flux density $\vec{v}$,

$$
\vec{j}=e \vec{v} \text {. }
$$

The second equation (11), combined with relation (12) and equation (15), makes it possible to find the current and thus, together with Maxwell's equations, completely determines the electromagnetic field in the plasma.

\section{FLUID APPROXIMATION}

The set of simplified equations derived from the kinetic equation, corresponds to the fluid approximation with regard for the thermal effects. The hydrodynamic velocity $\vec{u}$ is determined by the relation

$$
\vec{u}=\frac{\vec{v}}{N} \text {. }
$$

According to the definition (13), the quantity $\Delta_{i j}$, in the case of local equilibrium, reduces to

$$
\Delta_{i j}=\Delta \delta_{i j}, \quad \Delta=\frac{p}{m}, \quad p=N T,
$$

where $p$ is the plasma pressure, $T$ is the plasma temperature. Then equation (15) reduces to the usual thermal energy balance equation. Thus, (11) yields a set of hydrodynamic equations 


$$
\begin{aligned}
& \frac{\partial N}{\partial t}+\operatorname{div} N \vec{u}=0, \\
& \frac{\partial \vec{u}}{\partial t}+(\vec{u} \nabla) \vec{u}=-\frac{1}{m N} \nabla p+\frac{e}{m}\left(\vec{E}+\frac{1}{c}\left[\vec{u}, \vec{B}_{0}+\vec{B}\right]\right), \\
& \frac{\partial T}{\partial t}+(\vec{u} \nabla) T=-\frac{2}{3} T \operatorname{div} \vec{u} .
\end{aligned}
$$

The current $\vec{j}$ and the hydrodynamic velocity $\vec{u}$ satisfy the nonlinear relation $\vec{j}=e N \vec{u}$.

\section{THE LIMITING CASE OF COLD PLASMAS}

In the cold plasma limiting case, we can neglect the quantity $\Delta_{i j}$ or the pressure $(p=0)$ in the equation for the flux density $\vec{v}$ or for the hydrodynamic velocity $\vec{u}$. We also disregard the nonlinear terms and thus obtain the constitutive equation in the form $(3)$, where $\hat{x}(\omega)$ is the dielectric permittivity tensor of a cold magnetoactive plasma. We denote the incident wave frequency and wave vector by $\omega_{0}$ and $\vec{k}_{0}$, those of the scattered wave by $\omega$ and $\vec{k}$; the fluctuation frequency and wave vector are $\Delta \omega=\omega-\omega_{0}$ and $\vec{q}=\vec{k}-\vec{k}_{0}$. In what follows we label the quantities corresponding to the incident wave by the subscript 0 ; the fluctuation amplitudes are tilded. We treat both incident and scattered waves in the linear approximation.

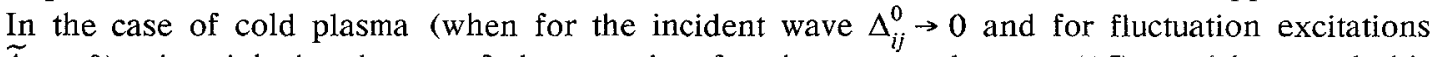
$\widetilde{\Delta}_{i j} \rightarrow 0$ ), the right-hand part of the equation for the scattered wave (15) vanishes, and this equation has a unique solution $\Delta_{i j}=0$. Hence, in the case under consideration, the current that induces scattered waves is determined by the equation for the flux density

$$
\frac{\partial \vec{v}}{\partial t}+\nabla \frac{\overrightarrow{v v}}{N}=\frac{e}{m}\left\{N \vec{E}+\frac{1}{c}\left[\vec{v}, \vec{B}_{0}+\vec{B}\right]\right\}
$$

Taking into account the nonlinear terms (which are, however, linear with respect to the incident wave and fluctuation amplitudes), we obtain an expression for the current that induces scattered waves, i.e.,

$$
\begin{aligned}
& \vec{J}_{\vec{k} \omega}=-i \frac{\omega}{n_{0}} \hat{x}(\omega)\left\{\vec{n}_{\vec{q} \Delta \omega} \vec{E}^{0}+n^{0} \overrightarrow{\widetilde{E}}_{\vec{q} \Delta \omega}+\right. \\
& \left.+\frac{1}{c}\left[\overrightarrow{\widetilde{v}}_{\vec{q} \Delta \omega}, \vec{B}^{0}\right]+\frac{1}{c}\left[\overrightarrow{\widetilde{v}}^{0}, \overrightarrow{\vec{B}}_{\vec{q} \Delta \omega}\right]-i \frac{m}{e n_{0}}\left(\left(\vec{k} \overrightarrow{\widetilde{v}}_{q \Delta \omega}\right) \vec{v}^{0}+\left(\vec{k} \vec{v}^{0}\right) \overrightarrow{\widetilde{v}}_{\vec{q} \Delta \omega}\right)\right\} .
\end{aligned}
$$

With the continuity equation being satisfied, equation (21) for the flux density $\vec{v}$ is equivalent to the equation for the hydrodynamic velocity $\vec{u}(19)$ with $p=0$.

This means that, in the cold plasma limiting case, the results of the fluid approximation reproduce the results obtained by the kinetic approach.

\section{IS THERE AMBIGUITY IN THE USE OF FLUID EQUATIONS?}

The direct use of equation (19) and current definition (20) results in different from expression for the scattered-waves-inducing current, i.e.,

$$
\begin{aligned}
& \vec{J}_{\vec{k} \omega}=-i \frac{\omega_{0}}{n_{0}}\left\{\widetilde{n}_{\vec{q} \Delta \omega} \hat{\varkappa}\left(\omega_{0}\right) \vec{E}^{0}+\frac{n_{0}}{\omega_{0}}\left(\overrightarrow{k_{0}} \hat{\varkappa}\left(\omega_{0}\right) \vec{E}^{0}\right) \vec{u}_{q \Delta \omega}+\right. \\
& \left.\left.+\frac{n_{0}}{\omega_{0}} \omega \hat{\varkappa}(\omega)\left[\frac{1}{c}\left(\widetilde{\vec{u}}_{\vec{q} \Delta \omega}, \vec{B}^{0}\right]+\left[\vec{u}^{0}, \overrightarrow{\widetilde{B}}_{\vec{q} \Delta \omega}\right]\right)-i \frac{m}{e}\left(\left(\vec{k}_{0} \widetilde{u}_{\vec{q} \Delta \omega}\right) \vec{u}^{0}+\left(\overrightarrow{q u}^{0}\right) \overrightarrow{\vec{u}}_{\vec{q} \Delta \omega}\right)\right]\right\} .
\end{aligned}
$$

In particular, the factors in the terms of equations (22) and (23) which describe wave scattering by electron density fluctuations, depend on different frequencies. The factor in (22) depends on the scattered wave frequency $\omega$, whereas the one in (23) depends on the incident wave frequency $\omega_{0}$. The difference between the expressions (22) and (23) which are derived from equivalent hydrodynamical equations (21) and (19), suggested an idea to the author of Ref. [13] that the fluid approach is inapplicable for the study of nonlinear processes in plasmas. Actually, 
however, expressions (22) and (23) are equivalent in the limiting case of cold plasma (this is just the case for which the hydrodynamic equations are applicable). For cold plasmas, one may disregard the thermal effects (i.e. thermal corrections in the dispersion wave equations) and the Langevin source in the constitutive equation for fluctuations.

We remind the reader that, when treating thermal fluctuations in a medium, one usually introduces in the constitutive equation an additional Langevin source $\vec{J}_{0}$ whose spectral distribution is assumed to be given, i.e.,

$$
\overrightarrow{\widetilde{J}}_{\vec{q} \tilde{\omega}}=-i \omega \hat{\varkappa}(\widetilde{\omega}) \overrightarrow{\vec{E}}_{\vec{q} \tilde{\omega}}+J_{0}
$$

This source is then employed to find the fluctuation spectral distributions for various quantities. We substitute $\vec{k}-\vec{k}_{0}$ for $\vec{q}$ in the last term within the curly braces in (23), and $\vec{k}-\vec{q}$ for $\vec{k}_{0}$ in the term last but one. Then, within the context of the relations $\overrightarrow{k_{0}} \vec{j}_{0}=-i \omega_{0}\left(\overrightarrow{k_{0}} \hat{\varkappa}\left(\omega_{0}\right) \vec{E}^{0}\right) \vec{E}^{0}$ and $\vec{q} \overrightarrow{\dot{j}_{\vec{q}} \Delta \omega}=\Delta \omega e \vec{n}_{\vec{q} \Delta \omega}$, and the identity $\hat{\varkappa}(\omega) \hat{\varkappa}^{-1}(\omega)=1$, we find that the expression for the current $(23)$ reduces to

$$
\begin{aligned}
& \vec{J}_{\vec{k} \omega}=-i \frac{\omega}{n_{0}} \hat{\varkappa}(\omega)\left\{\widetilde{n}_{q \Delta \omega} \frac{\omega_{0}}{\omega}\left(\hat{\varkappa}^{-1}(\omega)+4 \pi \frac{\omega \Delta \omega}{\omega_{p}^{2}}\right) \hat{\varkappa}\left(\omega_{0}\right) \vec{E}^{0}+i e n^{0} \frac{n_{0}}{\omega}\left(\hat{\varkappa}^{-1}(\omega)+4 \pi \frac{\omega \omega_{0}}{\omega_{p}^{2}}\right) \widetilde{\vec{u}}_{\vec{q} \Delta \omega}+\right. \\
& \left.+n_{0}\left[\frac{1}{c}\left(\left[\widetilde{\vec{u}}_{\vec{q} \Delta \omega}, \vec{B}^{0}\right]+\left[\vec{u}^{0}, \overrightarrow{\vec{B}}_{\vec{q} \Delta \omega}\right]\right)-i \frac{m}{e}\left(\left(\vec{k}_{\vec{u}} \vec{q}_{\vec{q} \Delta \omega}\right) \vec{u}^{0}+\left(\vec{k}^{0} \vec{u}^{0}\right) \widetilde{\vec{u}}_{\vec{q} \Delta \omega}\right)\right]\right\}
\end{aligned}
$$

The factor of the first term within the curly braces in the right-hand part of (25) takes the form $\hat{x}^{-1}(\omega)+4 \pi \frac{\omega \Delta \omega}{\omega_{p}^{2}}=\frac{\omega}{\omega_{0}} \hat{x}^{-1}\left(\omega_{0}\right)$, and therefore the first term in (25) reduces to the first term of (22). In a similar manner, we employ the relation $\hat{\varkappa}^{-1}(\omega)+4 \pi \frac{\omega \omega_{0}}{\omega_{p}^{2}}=\frac{\omega}{\Delta \omega} \hat{\varkappa}^{-1}(\Delta \omega)$ and rewrite $\quad$ (24) (in which $\vec{J}_{0}$ is neglected) as $\hat{\varkappa}^{-1}(\Delta \omega) \overrightarrow{\widetilde{J}}_{\vec{q} \Delta \omega}=-i \Delta \omega \overrightarrow{\widetilde{E}}_{\vec{q} \Delta \omega}$. Now we see that the second term in (25) reproduces the second term of (22). Thus we have shown that the expressions for the current that induces scattered waves, derived from the hydrodynamic equations (19) and (21), are equivalent provided we neglect the source $J_{0}$ in (24). In other words, in the limiting case of a cold plasma, derivation of the inducing current does not lead to any ambiguities.

\section{EFFECT OF THERMAL DISPERSION OF FLUCTUATIONS}

The phase velocities of the incident and scattered electromagnetic waves are much greater than the electron thermal velocity and hence thermal corrections to the dispersion of these waves may be disregarded. However, phase velocities of fluctuation excitations are of the same order of magnitude as the electron thermal velocity and therefore, under certain conditions, their thermal dispersion may considerably influence the wave scattering. If the distribution function $F$ is isotropic with respect to the velocity deviations from the mean vealue, then for fluctuation excitations we have $\widetilde{\Delta}_{i j}=\tilde{\Delta} \delta_{i j}$. As follows from the thermal balance equation,

$$
\tilde{\Delta}_{\vec{q} \Delta \omega}=\frac{5}{3} \frac{T}{m} \tilde{n}_{\vec{q} \Delta \omega}
$$

We disregard thermal dispersion corrections for the incident and scattered waves. At the same time, in the equation for the flux density $\vec{v}$ associated with the scattered wave, we allow for the thermal correction $\Delta_{i j}$ in the second moment. This correction is proportional to the temperature and, according to (15), is determined by the equation

$$
\Delta_{i j}(\vec{k}, \omega)-i \frac{\omega_{\mathrm{B}}}{\omega}\left(\epsilon_{i k l} \Delta_{j k}(\vec{k}, \omega)+\epsilon_{j k l} \Delta_{i k}(\vec{k}, \omega)\right)=S_{i j}(\vec{k}, \omega),
$$

where

$$
S_{i j}(\vec{k}, \omega)=\frac{5}{3} \frac{T}{m n_{0} \omega}\left(\vec{k}^{0}{ }^{0} \delta_{i j}+k_{i}^{0} v_{j}^{0}+k_{j}^{0} v_{i}^{0}\right) \tilde{n}_{\vec{q} \Delta \omega} .
$$


The equation for the scattered wave flux density contains only the scalar product $\hat{\Delta} \vec{k}$. In order to find it from the tensor equation $(27)$, we employ the simplified set of vector equations [16] for the quantities $\Delta \vec{k}, \Delta[\vec{k}, \vec{b}]$, and $\Delta \vec{b}$ ( $\vec{b}$ is the unit vector along the magnetic field $\vec{B}_{0}$ ). The solution of this set is given by

$$
\hat{\Delta}(\vec{k}, \omega) \vec{k}=-\frac{2 \pi}{\omega_{p}^{2}}\left\{\left(\omega-\omega_{\mathrm{B}}\right)^{2} \hat{\varkappa}\left(\omega-\omega_{\mathrm{B}}\right) \vec{Q}_{-}(\vec{k}, \omega)+\left(\omega+\omega_{\mathrm{B}}\right)^{2} \hat{\varkappa}\left(\omega+\omega_{\mathrm{B}} \vec{Q}_{+}(\vec{k}, \omega)\right\},\right.
$$

where the quantities $Q_{ \pm}(\omega)$ are defined by

$$
Q_{ \pm}(\vec{k}, \omega)=\frac{\omega}{\omega \pm \omega_{\mathrm{B}}}\left\{\hat{S}(\vec{k}, \omega) \vec{k} \mp 4 \pi \frac{\omega \omega_{\mathrm{B}}}{\omega_{p}^{2}}(\vec{k} \vec{b}) \hat{\varkappa}(\omega) \hat{S}(\vec{k}, \omega) \vec{b} \pm i \hat{S}(\vec{k}, \omega)[\vec{k}, \vec{b}]\right\}
$$

The additional inducing current, associated with the effect of electron thermal motion on the fluctuation dispersion, is described by the expression

$$
\left.\vec{J}_{\vec{k} \omega}^{T}=-\frac{m}{e n_{0}} \omega \hat{x}(t)\right) \hat{\Delta}(k, \omega) \vec{k} .
$$

Unlike the hydrodynamical current (22) which corresponds to zero plasma temperature, the thermal current (31), treated as a function of the scattered wave frequency, within the context of (29) has singularities at multiples of the cyclotron frequency, i.e., $\omega= \pm 2 \omega_{\mathrm{B}}$.

Therefore, if the incident wave frequency is close to twice the cyclotron frequency, the spectrum of incoherent scattering (associated with small frequency shifts) considerably depends on thermal dispersion of fluctuations. Singularities in the spectrum of electromagnetic wave scattering in magnetoactive plasmas were discovered in paper [17] by means of the kinetic approach.

We note that the linear set of fluid-approximation equations with regard for thermal effects yields thermal corrections to the wave dispersion which differ by factors from the analogous kinetic-approximation corrections. Therefore it is clear that the nonlinear set of hydrodynamic equations, obtained by truncating the infinite chain (11), provides only qualitative description of the scattering coefficient peculiarities asssociated with thermal effects. The quantitative study of thermal effects must be based on the kinetic description.

\section{Ackmowledgments}

The research, described in this publication was supported by The International Soros Science Education Program of International Renaissanse Foundation, grant SPU042060.

\section{References}

[1] Akhiezer A.I., Prohoba I.G. and Sitenko A.G., Soviet Physics JETP 6 (1958) 576-586.

[2] Akhiezer A.I., Akhiezer I.A. and Sitenko A.G., Soviet Physics JETP 14 (1962) 462-468.

[3] Sitenko A.G., Electromagnetic Fluctuations in Plasma (Academic,New York, 1967) 256 p.

[4] Akhiezer A.I., Akhiezer I.A., Polovin R.V., Sitenko A.G. and Stepanov K.N., Collective Oscillations in a Plasma (Pergamon, Oxford, 1967) $190 \mathrm{p}$.

[5] Sitenko A.G. and Kirochkin Yu.A., Soviet Phys. Usp. 9 (1966) 430-447.

[6] Salpeter E.E., Phys. Rev. 120 (1960) 1528 - 1535; Phys. Rev. 122 (1961) 1663-1674.

[7] Dougherty J.P. and Farley D.T., Proc. Roy. Soc. A259 (1960) $79-99$.

[8] Farley D.T., Dougherty J.P. and Barron D.W., Proc. Roy Soc. A263 (1961) 238-258.

[9] Aamodt R.E. and Russel D.A., Rev. Sci. Instrum. 61 (1990) 3211-3213.

[10] Chiu S.C., Phys. Fluids B3 (1991) 1374-1380.

[11] Vahala L., Vahala G. and Bretz N., Phys. Fluid B4 (1992) 619-629.

[12] Aamodt R.E. and Russel D.A., Nuclear Fusion 32 (1992) 745-755.

[13] Bindslev H., Plasma Phys. Control. Fusion 35 (1993) 1615-1640.

[14] Bindslev H., Физика плазмы 20 (1994) 39-41.

[15] Sitenko A.G., Plasma Phys. Control. Fusion 37 (1995) 163-167.

[16] Sosenko P.P. Electromagnetic wave scattering from plasma fluctuations (Göteborg, CTH - IEFT/PP - 1994) p. 19.

[17] Bindslev H., Plasma Phys. Control. Fusion 37 (1995) 169-176. 